Please send trade news information and illustrations to Arveen Bajaj at the BDJ, 64 Wimpole Street, London W1G 8YS. Trade news is supplied as a service to the reader and does not imply endorsement by the BDJ. Normal and prudent research should be exercised before purchase of use of any product mentioned.

\section{TRADE NEWS \\ WHAT'S NEW}

\section{Fun oral hygiene}

The British Dental Health Foundation has a range of brightly coloured hygiene demonstrators to help get the oral health message across. The brightly coloured animals from the six-puppet range include Brusher Boa and Mustang Max, Bugsy Malone, Freddy Flossisauras, Doogan the Dog and Alexander the Alligator. The demonstrators can capture the imagination of children in order for the oral health educator to demonstrate brushing and flossing techniques, the organisation claims. They include a hidden water squirter and large toothbrush.

Reader response number 50

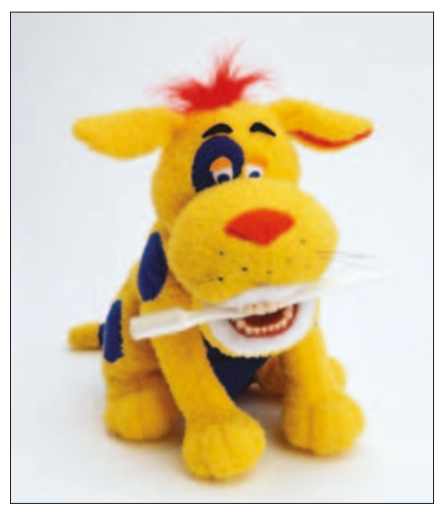

\section{Christmas prize draw}

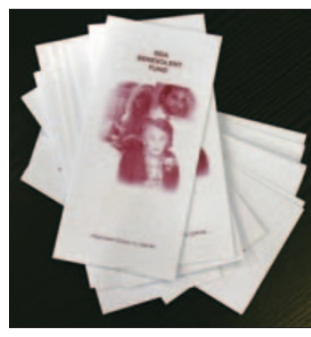

The Benevolent Fund offers unconditional help in time of need to any dentist who has ever been registered in the UK, and to the dentist's family.

The Fund helps with special grants at Christmas so its beneficiaries can join in the festivities with everyone else and is running its 31st Christmas Draw.

The first prize is $£ 6,000$ in cash, the second prize is $£ 500$ in holiday vouchers while the third prize is $£ 250$ in holiday vouchers.

The prize draw will take place on 29 January 2005. Please contact the Benevolent Fund to enter.

Reader response number 52

\title{
New dye launched
}

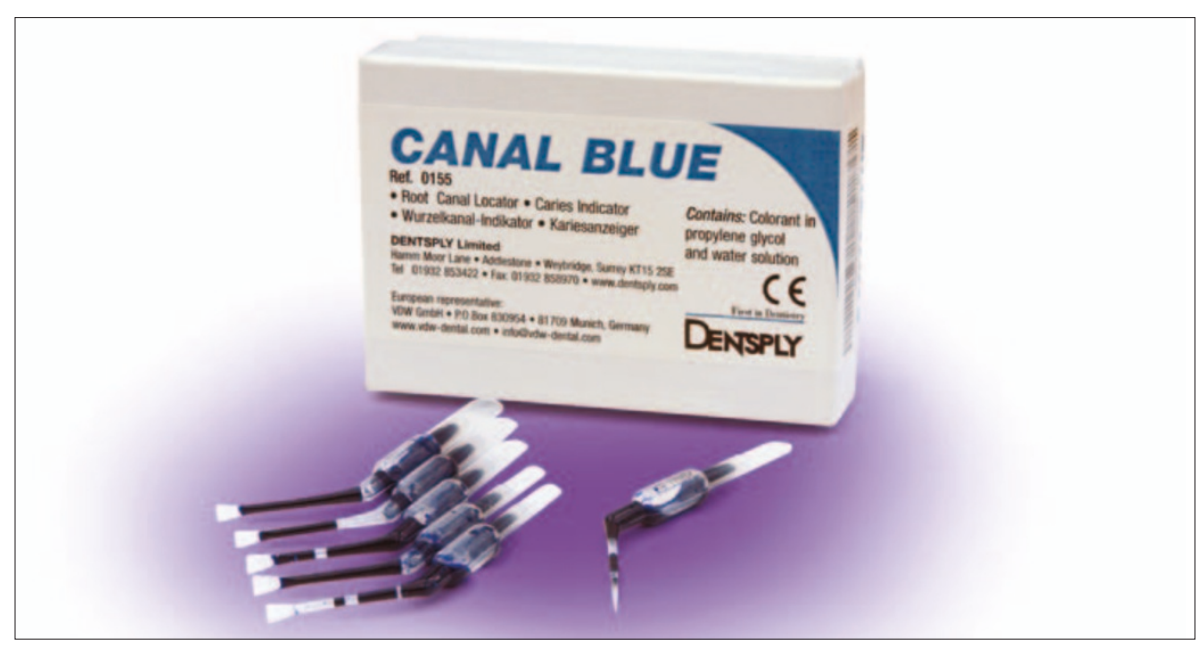

Dentsply have recently launched Canal Blue, a blue vegetable dye used in endodontic procedures to easily identify root canal orifices, which also allows for the identification of hairline cracks and carious dentine. Twenty-five pipettes of Canal Blue come packaged in each box. These single-dose, disposable pipettes, ensure no risk of cross contamination between patients, Dentsply claim.
The product can be used by cutting off the end of the pipette and coating the floor of the pulp chamber with the contents of the pipette. After waiting ten seconds, rinsing and drying, the canal orifices will be identified as simple blue dots. It can also be used in the same way to detect hairline cracks and infected carious dentine, without colouring healthy dentine. Reader response number 51

\section{New booklet for teenagers launched}

Bonjela has launched a new, free patient booklet, 'Straight Talk', for parents of teenagers to help them prepare for life with an orthodontic device.

It is a handy A5 booklet providing readers with lots of easy-to-read, jargon-free information such as how an orthodontic appliance actually works and the process that the wearer will go through until their fixture is removed.

It also provides handy tips on keeping removable appliances and retainers sparkling clean with cleaning tips including the correct way to floss and good brushing techniques. Answers are given to questions such as what to expect when their brace is tightened and how to help brace sores to heal. Bonjela is offering 'Straight Talk' to dental professionals free of charge for their waiting rooms. Copies can be ordered by calling the Bonjela Medical Information Unit on 0500455456.

Reader response number 53

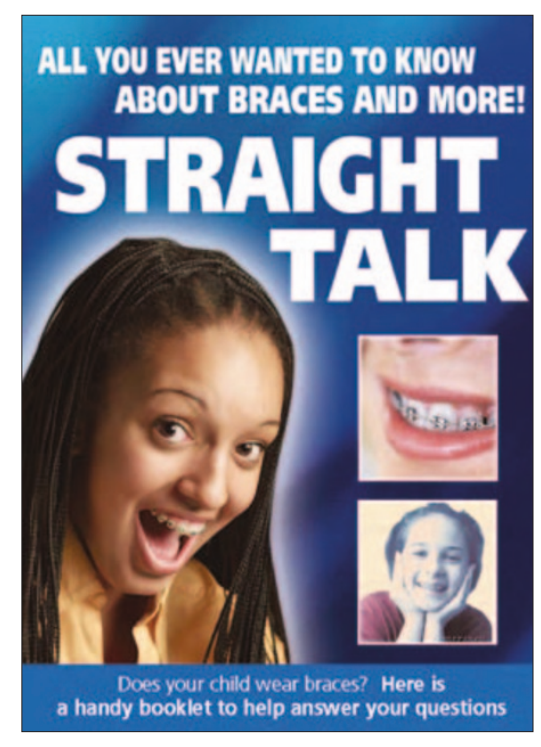



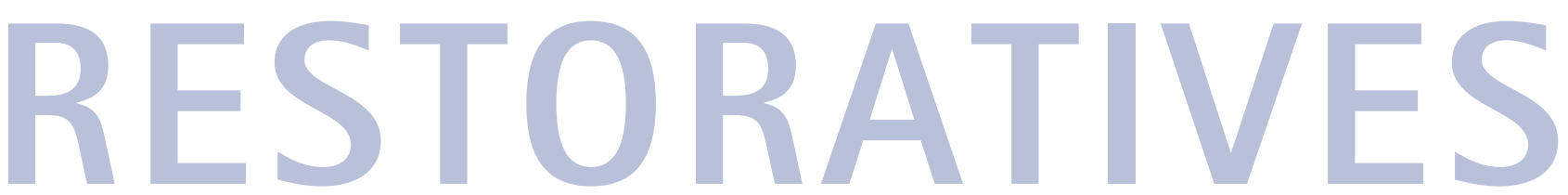

\section{Range of casting alloys}

Metalor's range of dental alloys includes the Medium Gold content alloy which contains between $60 \%$ and $66 \%$ gold. It claims that the alloys are consistent in quality, immensely durable, reliable and strong and can be used for crowns, bridges and implants.

Its Low Gold Content alloys contain a maximum 41\% gold and appear pale yellow or white in appearance.

They can be used for full or partial dentures, posts and cores, crowns and bridge work. Products in this range include the Yellow Special Type III which is a hard, pale yellow, multipurpose gold and the White XL Type IV, which is an extra hard white multipurpose gold.

Reader response number 54

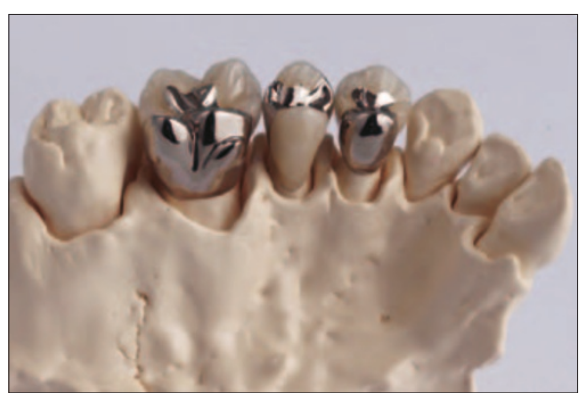

\section{Odourless tablet}

A-dec's waterline maintenance product ICX comes in the form of an odourless effervescent tablet. As the tablet dissolves, it releases ingredients into the water that help prevent the build-up of deposits and contaminants. ICX tablets come in self-dispensing packets for either 0.7-litre or 2litre water bottles.

\section{Reader response number 56}

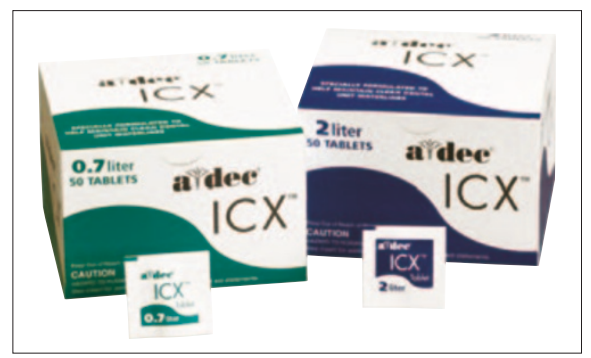

\section{Futuristic restoratives}

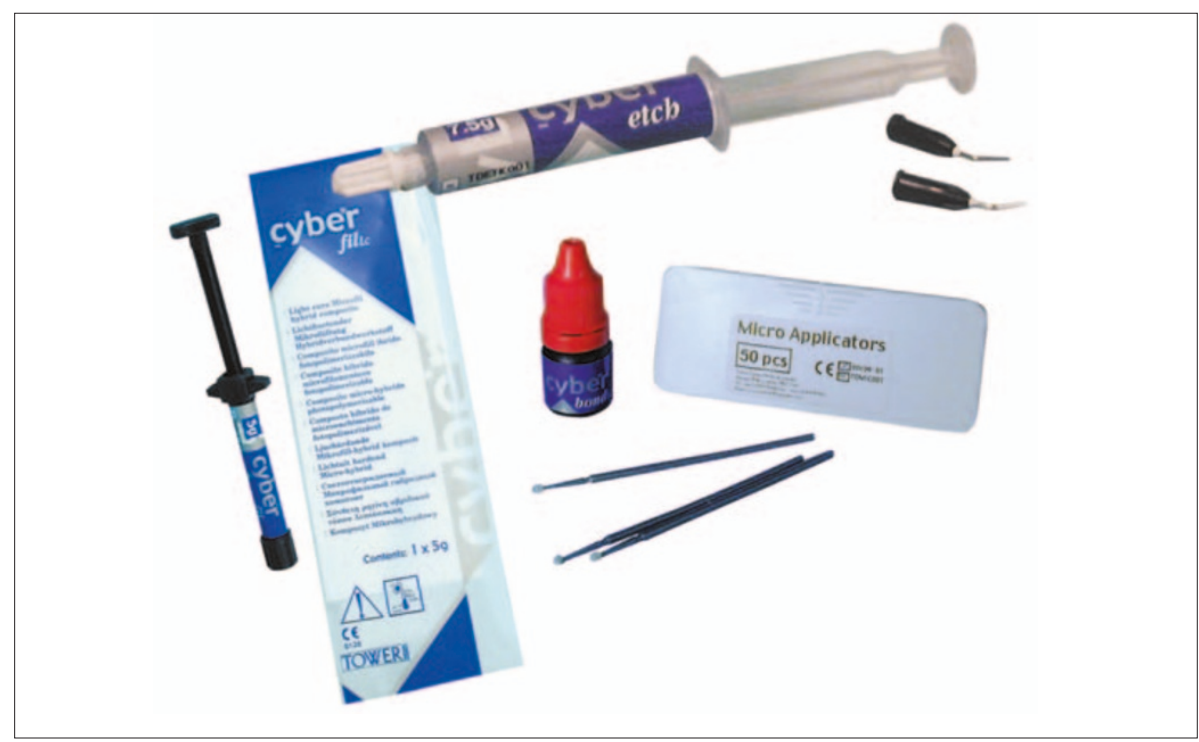

The Cyber range from Tower Dental has been designed to bring together the latest materials and techniques at a cost-effective price, according to the company.

CyberFil is a microfil hybrid composite for anterior and posterior restorations. Strongly radiopaque and light curing, it possesses good colour stability and polishes to a glossy and durable finish.

The product is specially designed for use with CyberEtch gel and CyberBond and is also compatible with equivalent products from other major manufacturers. CyberEtch is a blue etch gel contain-

\section{Quick set}

Kemdent's Diamond range of advanced restorative cements is tough, durable and develops a compressive strength of 350 Mpa, the company claims. The latest additions to the range include Diamond Snappy which is specially designed for fillings in children's teeth,

Diamond Rapid Set Capsules which are quick and easy to use with a waterproof snap set and Diamond Corem the strongest product in the Diamond range. It is specially designed for core build-up, has a rapid snap set, saliva resistance and adheres to dentine and enamel. In addition, in Kemdent's Win- ing 37\% phosphoric acid. Coloured blue for easy identification on application and removal, it can be used for the etching of tooth enamel and as a dentine conditioner, prior to bonding direct composite restorations.

Lastly, CyberBond is an all-purpose, light curing, single component primer and dentine/enamel adhesive which is also hydrophilic and can be used for cavity sealing before placing amalgam restorations and for cementing repair work.

Reader response number 55

ter Sale you can buy any two products from the Diamond restorative cements range and receive a 10\% discount.

Reader response number 57

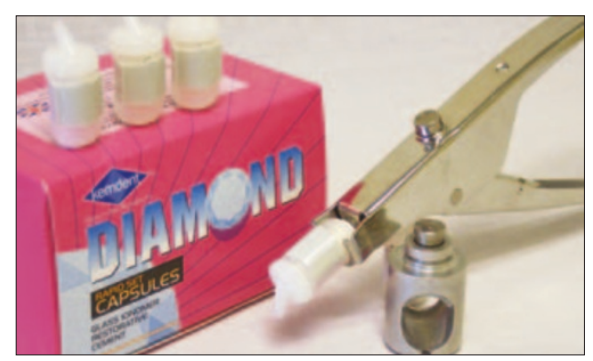




\section{Natural match}

The Vita Physiodens is a premium acrylic tooth which in its shape and size is identical with the average design of natural teeth, Vita clams.

The range covers the field of partial and full dentures and the material used is a special acrylate enriched with inorganic fillers which ensure durable restorations and prevents discolouration.

The anteriors have a smooth transition from the tooth crown towards the tooth neck which supports the natural design of papillae and gingivae while individual incisal edges enhance the appearance of the tooth.

The Vita Physiodens tooth is available in the systems Vita System 3D-Master (26 shades) and Vitapan classical (15 shades).

Reader response number 58

\section{Permanent bond}

Chemfil Superior is a glass-ionomer which its makers claim can establish a true, permanent bond to hydroxyapatite even under the unfavourable, moist conditions of cavities close to the gingival margin. In addition, it allows dentists

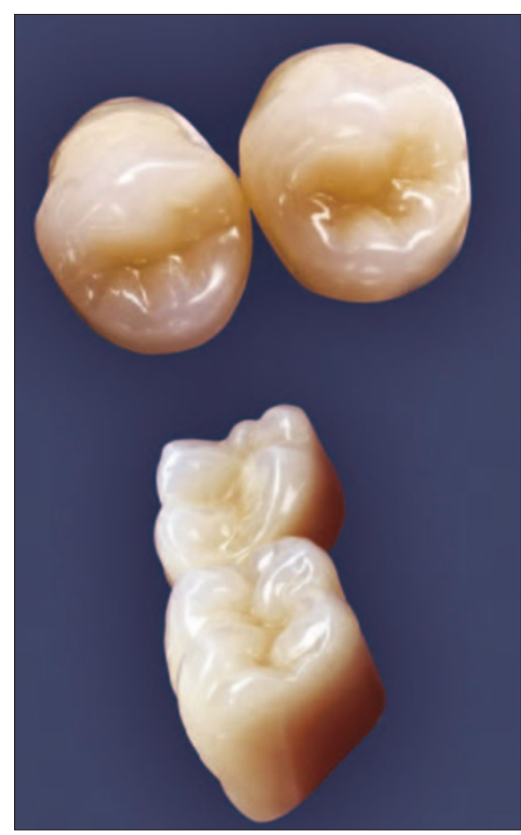

enough time to work before a rapid set, and can be used in cavities with margins in dentine or cavities close to the gingival margin, where moisture control is not possible.

Reader response number 59

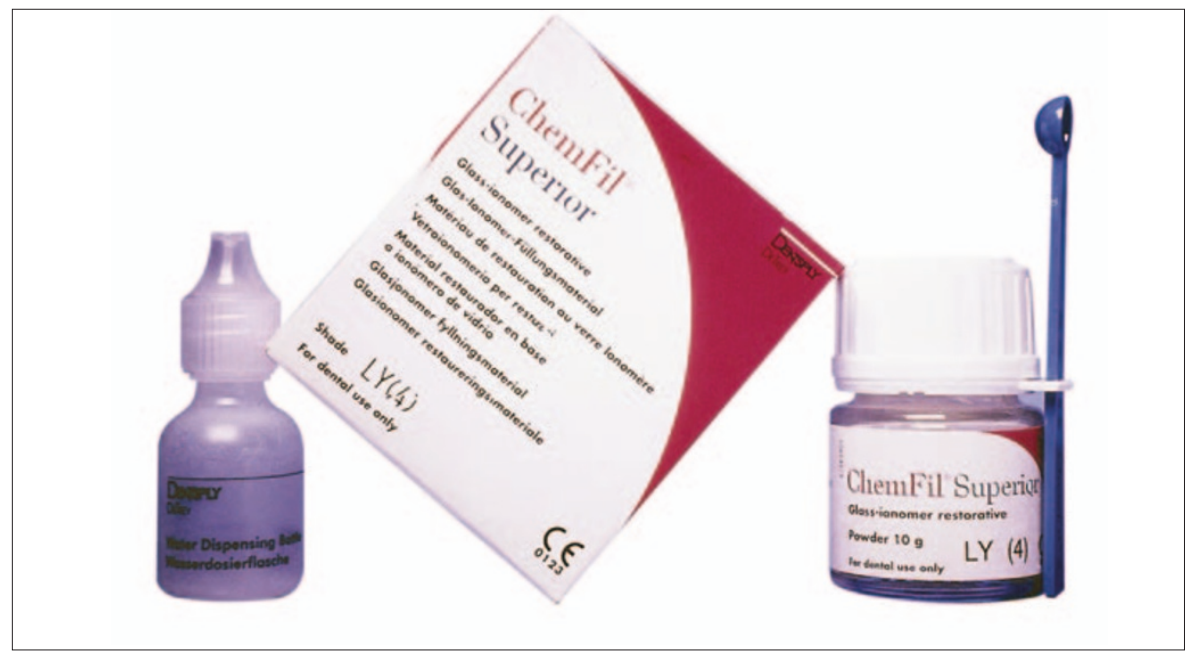

\section{Drill with a twist}

Busch \& Co have developed the special twist drill 203HM to set drillings for uptakes of retaining or positioning pins for attachments.

The twist drill is made out of finest grain carbide. The new design of the carbide twist drill improves the discharge of chips and allows holes to be drilled accurately in a large variety of alloys, including those which are difficult to cut, the manufacturers claim.

The drills are available in four different diameters with a short handpiece shank $(34 \mathrm{~mm})$ for cutting.

Reader response number 60

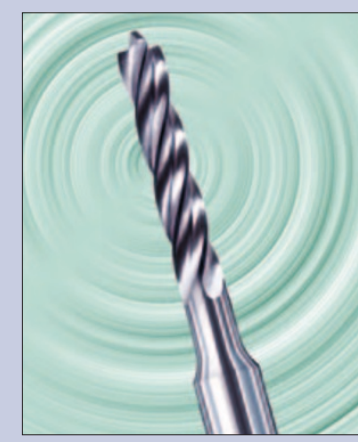

